\title{
Notes on homogeneous almost Hermitian manifolds
}

\author{
By Kouei Sekigawa \\ (Received September 9, 1977; Revised November 11, 1977)
}

\section{Introduction}

The object of this paper is to characterize homogeneous almost Hermitian manifolds (i. e., almost Hermitian manifolds with transitive automorphism groups) in terms of the behavior of their curvature tensors and structure tensors under parallel translations. In [1], W. Ambrose and I. M. Singer have characterized homogeneous Riemannian manifolds in terms of the behavior of their curvature tensors under the parallel translations. They have proved the following

TheOREM A. Let $(M,\langle\rangle$,$) be a homogeneous Riemannian manifold.$ Then, there exists a skew-symmetric tensor field $T$ of type $(1,2)$ on $M$ satisfying

$$
\begin{array}{ll}
\text { (A) } & \nabla_{X} R=T(X) \cdot R, \\
\text { (B) } & \nabla_{X} T=T(X) \cdot T, \quad \text { for any tangent vector } X \in T_{x}(M), x \in M .
\end{array}
$$

Conversely, if a connected, simply connected complete Riemannian manifold $(M,\langle\rangle$,$) admits a skew-symmetric tensor field T$ of type $(1,2)$ on $M$ satisfying $(A)$ and $(B)$, then $(M,\langle\rangle$,$) is a homogeneous Riemannian manifold.$ In this note, we shall prove the following

THEOREM B. Let $(M, J,\langle\rangle$,$) be a homogeneous almost Hermitian$ manifold. Then, there exists a skew-symmetric tensor field $T$ of type $(1,2)$ satisfying $(A),(B)$ in Theorem $A$, and furthermore

(C) $\quad \nabla_{X} J=T(X) \cdot J$, for any tangent vector $X$.

Conversely, if a connected, simply connected, complete almost Hermitian manifold $(M, J,\langle\rangle$,$) admits a skew-symmetric tensor field T$ of type $(2,1)$ satisfying $(A),(B)$ and $(C)$, then $(M, J,\langle\rangle$,$) is a homogeneous almost Her-$ mitian manifold.

By slight modification of the proof of Theorem A, we may prove Theorem B (cf. §3, §4). As a result of Theorem B, we have the following wellknown result. If $(M, J,\langle\rangle$,$) is a connected, simply connected Hermitian$ symmetric space, then it is homogeneous almost Hermitian manifold. 
REMARK. Let $(M,\langle\rangle),((M, J,\langle\rangle$,$) , resp. )$ be a homogeneous Riemannian manifold (a homogeneous almost Hermitian manifold, resp.). Then, by Theorem A (Theorem B, resp.), there exists a skew-symmetric tensor field $T$ of type $(1,2)$ on $M$ satisfying (A) and (B) (and furthermore, (C), resp.). However, such a tensor field $T$ does not necessarily exist uniquely on $M$.

This corresponds to the fact that the expression of $M$ as left coset space is not necessarily determined uniquely. So, in this case, we shall call that $(M,\langle\rangle),((M, J,\langle\rangle$,$) , resp. )$ has homogeneous structure for $T$. In $\S 5$, we shall state some related results of Theorem B.

\section{Preliminaries}

Let $L(M)$ be the linear frame bundle over a connected $m$-dimensional $C^{\infty}$-manifold $M$ with the projection $\pi$. And let $(M,\langle\rangle),((M, J,\langle\rangle$,$) , resp.)$ be a connected $m$-dimensional Riemannian manifold $(m(=2 n)$-dimensional almost Hermitian manifold, resp.), and $O(M)=O(M,\langle\rangle),(U(M)=U(M, J$, $\langle$,$\rangle ), resp.) be the orthonormal frame bundle (the unitary frame bundle,$ resp.) over $M$ with respect to the Riemannian structure $\langle$,$\rangle (almost Her-$ mitian structure $(J,\langle\rangle$,$) , resp.) with the projection \pi=\left.\pi\right|_{o(M)}\left(\pi=\left.\pi\right|_{U(M)}\right.$, resp.). Let $\boldsymbol{R}^{m}$ be an $m$-dimensional real number space and $\left(\boldsymbol{v}_{i}\right) ; \boldsymbol{v}_{i}=(0, \cdots, \stackrel{i}{1}, 0, \cdots$, $0) \in \boldsymbol{R}^{m}, i=1,2, \cdots, m$, be the canonical basis for $\boldsymbol{R}^{m}$. Then, each $b=(x$; $\left.e_{1}, \cdots, e_{m}\right) \in O(M)$ can be regarded as a linear isomorphism from $\boldsymbol{R}^{m}$ onto $T_{x}(M)$ such that $b\left(v_{i}\right)=e_{i}, i=1,2, \cdots, m$. Let $O(m)((\boldsymbol{R} U(n), m=2 n$, resp. $)$ be the orthogonal group of degree $m$ (the real representation of the unitary group of degree $n$, resp.), and $o(m)(\boldsymbol{R} u(n)$ or $u(n)$ simply, resp.) be the Lie algebra. Now, we define linear mappings $f_{i j}(i<j)$ on $\boldsymbol{R}^{m}$ by $f_{i j}\left(v_{i}\right)=-\boldsymbol{v}_{j}$, $f_{i j}\left(v_{j}\right)=v_{i}$, and $f_{i j}\left(v_{k}\right)=0$, for $k \neq i, j$. Then, $\left(f_{i j}\right)(i<j)$ is a basis for $o(m)$. Furthermore, we put $f_{j i}=-f_{i j}(i<j)$. Let $F_{i j}$ be the fundamental vector fields on $O(M)$ corresponding to $f_{i j} \in o(m), i, j=1,2, \cdots, m$, and $\mathscr{F}$ be the Lie algebra consisting of all vector fields on $O(M)$ of the form, $\sum_{j<k} b_{j k} F_{j k}$, $b_{j k} \in \boldsymbol{R}$. Now, let $\boldsymbol{\nabla}$ be the Riemannian connection on $M$ with respect to the Riemannian metric $\langle$,$\rangle , and \omega$ be the corresponding connection 1 -form on $O(M)$. Then, we may associate with each $v \in \boldsymbol{R}^{m}$ the standard horizontal vector field (or the basic vector field) $E(v)$ on $O(M)$ with respect to $\omega$. We put $E_{i}=E\left(v_{i}\right), i=1,2, \cdots, m$. Then, $\left(E_{i}\right)$ makes a basis for horizontal subspace at each $b \in O(M)$. Let $\mathcal{E}$ be the $m$-dimensional vector space consisting of all vector fields on $O(M)$ of the form, $\sum_{i} a_{i} E_{i}, a_{i} \in \boldsymbol{R}$. Then, $\mathscr{F}$ acts on $\mathcal{E}$ by

$$
\left[F_{i j}, E(v)\right]=E\left(f_{i j} v\right), \quad i, j=1,2, \cdots, m
$$


Next, consider an $m(=2 n)$-dimensional almost Hermitian mạnifold $(M$, $J,\langle\rangle$,$) . We put$

$$
\begin{aligned}
B_{i j} & =(1 / \sqrt{2})\left(F_{i j}+F_{n+i n+j}\right), & & (i<j), \\
C_{i j} & =(1 / \sqrt{2})\left(F_{i n+j}+F_{j n+i}\right), & & (i \neq j), \\
C_{i} & =F_{i n+i} . & &
\end{aligned}
$$

Then, $\left(B_{i j}(i<j), C_{i j}(i \neq j), C_{i}\right)$ is a basis of the vertical subspace at each point $\boldsymbol{b} \in U(M)=U(M, J,\langle\rangle$,$) . Of course, U(M)$ can be regarded as a principal subbundle of $O(M)$ which is the orthonormal frame bundle over $M$ with respect to the Riemannian structure.

Next, if $S$ is a linear transformation on $T_{x}(M), x \in M$, then it gives rise to a $g l(m, \boldsymbol{R})$-valued function $\sigma(S)$ on $\pi^{-1}(x)$ by

$$
S e_{j}=\sum_{i=1}^{m}\left(\sigma_{i j}(S)\right)(b) e_{i}, \quad \text { for } b=\left(x ; e_{1}, \cdots, e_{m}\right) \in L(M) .
$$

Then, we may see that $\sigma(S)$ is equivariant (or of $\operatorname{ad}(O(m))$-type). Conversely, any equivariant $g l(m, \boldsymbol{R})$-valued function $\sigma$ on $\pi^{-1}(x)$ is such a $\sigma(S)$ for a unique linear transformation $S$ on $T_{x}(M)$.

Now, let $T$ be a skew-symmetric tensor field of type $(1,2)$ which assigns to each point a skew-symmetric linear transformation $T(X)$ on $T_{x}(M)$ for each $X \in T_{x}(M)$. Then, we can point out that such a $T$ gives rise to a unique equivariant horizontal $o(m)$-valued 1-form $\tau$ on $O(M)$, and there is a 1-1 correspondence between the set of all such $T$ 's and the set of all such $\tau$ 's (cf. [1]). If $T$ and $\tau$ correspond, then $T$ is of class $C^{\infty}$ if and only if $\tau$ is of class $\mathrm{C}^{\infty}$. Next, we shall consider the covariant derivatives, $\nabla_{X} K$, $\left(\nabla_{X} T\right)(Y)$ ans $\left(\nabla_{X} R\right)(Y, Z)$, etc.; where $K$ is any tensor field of type $(1,1)$, $T$ a skew-symmetric tensor field, of type $(1,2)$, and $R$ the curvature tensor. We first consider $\nabla_{X} K$, for $X \in T_{x}(M), X \neq 0$. Let $\gamma$ be the geodesic in $M$ tangent to $X$ at $x$, and parametrized so that $\gamma(0)=x$. Let $P_{t}$ denote the linear transformation of parallel translation along $\gamma$ from $x$ to $\gamma(t)$. We get a 1-parameter family of linear transformations $S_{t}$ on $T_{x}(M)$ by defining $S_{t}=P_{t}^{-1} K_{r(t)} P_{t}$. Then, $\nabla_{X} K$ is given by the derivative of $S_{t}$ at $t=0$, by the definition. That is to say, if $\left(e_{i}\right)=\left(e_{1}, \cdots, e_{m}\right)$ is an orthonormal frame at $x$ and $\left(a_{i j}(t)\right)$ is the matrix of $S_{t}$ with respect to this $\left(e_{i}\right)$, then $\nabla_{X} K$ is the linear transformation on $T_{x}(M)$ whose matrix with respect to $\left(e_{i}\right)$ is $\left(a_{i j}(0)\right)$. To interpret this in terms of $\tau$, let $b$ be the point of $O(M), b=\left(x ; e_{1}, \cdots\right.$, $\left.\boldsymbol{e}_{m}\right)$, and $\left(e_{i}(t)\right)=\left(e_{1}(t), \cdots, e_{m}(t)\right)$ be the parallel translates of the $\left(e_{i}\right)$ along $\gamma$ and $\bar{\gamma}$ be the curve in $O(M)$ defined by $\bar{\gamma}(t)=\left(\gamma(t) ; e_{1}(t), \cdots, e_{m}(t)\right)$. Then, $a_{i j}(t)=$ $\left\langle S_{t} e_{i}, e_{j}\right\rangle=\left\langle P_{t}^{-1} K_{r(t)} P_{t} e_{i}, e_{j}\right\rangle=\left\langle K_{r(t)} P_{t} e_{i}, P_{t} e_{j}\right\rangle=\left\langle K_{r(t)} e_{i}(t), e_{j}(t)\right\rangle=\sigma_{i j}\left(K_{r(t)}\right)(\bar{\gamma}(t))$. 
Let $E \in \mathcal{E}$ with $d \pi(E(b))=X$. Then, by the above equation, we have

$$
\sigma\left(\nabla_{X} K\right)(b)=E(b) \sigma(K) \text {. }
$$

Similarly, $\left(\nabla_{X} T\right)(Y)$ and $\left(\nabla_{X} R\right)(Y, Z)$, for $X, Y, Z \in T_{x}(M), X \neq 0$, are given respectively as follows (cf. [1]) :

$$
\begin{aligned}
& \sigma\left(\left(\nabla_{X} T\right)(Y)\right)(b)=E(b) \tau(E), \\
& \sigma\left(\left(\nabla_{X} R\right)(Y, Z)\right)(b)=E(b) \Omega\left(E^{\prime}, E^{\prime \prime}\right),
\end{aligned}
$$

where $E, E^{\prime}, E^{\prime \prime} \in \mathcal{E}$ such that $d \pi(E(b))=X, d \pi\left(E^{\prime}(b)\right)=Y, d \pi\left(E^{\prime \prime}(b)\right)=Z$, and $\Omega$ denotes the curvature form with respect to the Riemannian connection $\omega$ on $O(M)$.

In general, the following is well known (cf. [1], [3]).

LEMMA 2.1. If $\theta$ is an equivariant horizontal $C^{\infty} p(\geqq 0)$-form on $O(M)$ with the values in $o(m)$, then

$$
d \theta=(-1)^{p}[\theta, \omega]+D \theta,
$$

where $D \theta=d \theta \circ H, H$ denoting the projection onto the horizontal subspace. In particular, if $\theta=\Omega$, since $D \Omega=0$ by the second Bianchi identity, we have

$$
d \Omega=[\Omega, \omega] .
$$

\section{The first half of Theorem $B$.}

In this section, we assume that $(M, J,\langle\rangle$,$) is connected, homogeneous$ almost Hermitian manifold. We define here a certain equivariant horizontal $C^{\infty} 1$-form $\tau$, then show that the associated $T$ satisfies the three conditions (A), (B) and (C) in Theorem B by showing that $\tau$ has corresponding properties. Let $G$ be a transitive Lie group of automorphisms of $(M, J,\langle\rangle$,$) .$ Then $G$ acts on $U(M)$ if we define

$$
\begin{aligned}
g(x ; & \left.e_{1}, \cdots, e_{n}, J e_{1}, \cdots, J e_{n}\right) \\
& =\left(g x ;(d g) e_{1}, \cdots,(d g) e_{n},(d g) J e_{1}, \cdots,(d g) J e_{n}\right) \\
& =\left(g x ;(d g) e_{1}, \cdots,(d g) e_{n}, J(d g) e_{1}, \cdots, J(d g) e_{n}\right), \\
& \quad \text { for any } g \in G .
\end{aligned}
$$

We put on $O(M)$ (which is the orthonormal frame bundle with respect to the Riemannian structure of $(M, J,\langle\rangle)$,$) the Riemannian structure in which$ at each $b \in O(M)$ the $E_{i}(b)$ and $F_{j k}(b)(j<k)$ are an orthonormal basis of $T_{b}(O(M))$. Furthermore, we put on $U(M)$ the Riemannian structure which is induced from the above Riemannian structure on $O(M)$. Then, $B_{j k}(b)$ 
$(j<k), C_{j k}(b)(j \neq k), C_{i}(b)$, and $E_{i}(b)$ are an orthonormal basis of $T_{b}(U(M))$, $b \in U(M)$. Under the above action, $G$ is also group of isometries of $U(M)$. This is trivial from the fact that any $g \in G$ preserves everything naturally attached to the almost Hermitian structure of $(M, J,\langle\rangle$,$) . Since M$ is connected, it is well known that an automorphism of $(M, J,\langle\rangle$,$) is determined$ by it and its differential at a single point. Hence, for any $b \in U(M)$, the mapping $L_{b}$ of $G$ into $U(M)$ defined by $L_{b}(g)=g b$, is a $1: 1$ mapping of $G$ into $U(M)$. It is also well known that $L_{b}$ is of class $C^{\infty}$ and that $d L_{b}$ is nonsingular everywhere and hence, $L_{b}(G)$ is a submanifold of $U(M)$. Let $T_{e}(G)$ be the tangent space at the identity $e$. and $P_{b}=d L_{b}\left(T_{e}(G)\right)$. We then define a subspace $Q_{b}$ by

$$
Q_{b}=P_{b} \cap\left(P_{b} \cap V_{b}\right)^{\perp},
$$

where $V_{b}$ is the vertical subspace at $b$ and " $\perp$ " denotes the orthogonal complement with respect to the Riemannian metric defined above on $U(M)$. Then, we can show that the distribution $Q$ defines a connection on $U(M)$ (cf. [1]). Next, we can extend this connection on $U(M)$ to the one of $O(M)$ by the natural fashion. Let $\phi$ be the connection 1 -form corresponding to the connection $Q$ on $O(M)$. And we define $\tau$ by $\tau=\omega-\phi$. Then, we can easily see that $\tau$ is equivariant and horizontal. In terms of the connection $Q$ every $\xi \in T_{b}(O(M))$ can be decomposed into a $Q$-horizontal and a vertical vector, and we denote these components by $Q \xi$ and $W \xi$, so $\tau=Q \xi+W \xi$, where $W \xi$ is vertical and $Q \xi \in Q_{b}, \xi \in T_{b}(O(M)), b \in O(M)$. We note the following (cf. [1])

$$
\tau(E)=\omega(Q E)=-\omega(W E), \quad \text { if } E \in \mathcal{E} .
$$

Now, we can show the following (cf. [1])

Lemma 3. 1. If $b \in U(M)$ and $E, E^{\prime} \in \mathcal{E}$, then the functions, $\sigma(J), \Omega\left(E, E^{\prime}\right)$ and $\tau(E)$ are constant on $L_{b}(G)$.

We now consider the meaning of (2.5) and (2.6) in terms of vector fields in $\mathcal{E}$ and $\mathscr{F}$. Then, by Lemma 3.1, we have the followings.

$$
F \sigma(J)=d(\sigma(J))(F)=[\sigma(J), \omega(F)],
$$

and furthermore (cf. [1])

$$
\begin{aligned}
& F \tau(E)=\tau([F, e])+[\omega(F), \tau(E)], \\
& F \Omega\left(E, E^{\prime}\right)=-\Omega\left([E, F], E^{\prime}\right)+\Omega\left(\left[E^{\prime}, F\right], E\right)+\left[\Omega\left(E, E^{\prime}\right), \omega(F)\right],
\end{aligned}
$$

for all $E, E^{\prime} \in \mathcal{E}, F \in \mathscr{H}$. 
Since $(M, J,\langle\rangle$,$) is a homogeneous Riemannian manifold with respect to$ the Riemannian structure, by (2.3), (2.4), (3.3), (3.4), Lemma 3.1, and the arguments in [1], we see that (A) and (B) in Theorem $\mathrm{A}$ hold for the tensor field $T$ corresponding to $\tau$.

Furthermore, by Lemma 3.1, we have

$$
E(b) \sigma(J)=(W E)(b) \sigma(J)=F(b) \sigma(J),
$$

where $F(b)=(W E)(b) \in V_{b}$.

Thus, by (3.2) and (3.5), we have

$$
\begin{aligned}
E(b) \sigma(J) & =[\sigma(J)(b), \omega(F(b))] \\
& =-[\sigma(J)(b), \omega((Q E)(b))] \\
& =[\omega((Q E)(b)), \sigma(J)(b)] \\
& =[\tau(E)(b), \sigma(J)(b)] \\
& =[\sigma(T(X))(b), \sigma(J)(b)] \\
& =\sigma([T(X),(J)])(b),
\end{aligned}
$$

where $d \pi(E(b))=X$.

Thus, from (2.2) with $K=J$, and (3.6), we have

$$
\nabla_{X} J=[T(X), J] \text {. }
$$

(3.7) is nothing but $(\mathrm{C})$ in Theorem $\mathrm{B}$. This completes the proof of the first half of Theorem B.

\section{The latter half of Theorem $B$.}

In this section, we assume that $(M, J,\langle\rangle$,$) is a connected, simply con-$ nected, complete almost Hermitian manifold with a skew-symmetric tensor field $T$ of type $(1,2)$ satisfying the conditions, (A), (B) and (C) in the introduction. For this $T$, we can construct $\tau$ which is the associated horizontal, equivariant $C^{\infty} 1$-form on $O(M)$. Furthermore, using this $\tau$, we can define a connection 1 -form, $\phi$ by $\phi=\omega-\tau$. Let $Q_{b}$ be the horizontal subspace of $T_{b}(O(M))$ with with respect to $\phi$, i. e., $Q_{b}=\left\{\xi \in T_{b}(O(M)) ; \phi(\xi)=0\right\}$. We now fix a point $b_{0}=\left(x_{0} ; e_{1}^{0}, \cdots, e_{n}^{0}, J e_{1}^{0}, \cdots, J e_{n}^{0}\right) \in U(M)$ and a subset $G$ of $O(M)$ to be the set of all points in $O(M)$ that can be joined to $b_{0}$ by broken $C^{\infty}$ $Q$-horizontal curves in $O(M)$. Then, $G$ is a principal subbundle of $O(M)$. Furthermore, we can show the following by reversing the arguments in 
$\S 3$ (cf. [1]).

LEMMA 4.1. Under our assumptions, the functions, $\sigma(J), \tau(E)$ and $\Omega\left(E, E^{\prime}\right)$ are constant on $G$, for all $E, E^{\prime} \in \mathcal{E}$.

Furthermore, according to [1], we can show that $G$ has a Lie group structure and acts as a transitive group of isometries of $(M, J,\langle\rangle$,$) by b^{\prime}(x)=$ $\pi\left(b^{\prime} b\right), b, b^{\prime} \in G, \pi(b)=x$. When we regard $b^{\prime} \in G$ as an isometry of $(M$, $J,\langle\rangle$,$) , we denote it by g^{\prime}=g\left(b^{\prime}\right)$.

Since

$$
\sigma(J)\left(b_{0}\right)=\left(\begin{array}{rr}
0 & I_{n} \\
-I_{n} & 0
\end{array}\right),
$$

by Lemma 4.1, we see that every $b \in G$ takes of the form, $b=\left(x ; e_{1}, \cdots, e_{n}\right.$, $\left.J e_{1}, \cdots, J e_{n}\right)$, where $I_{n}$ denotes the unit matrix of degree $n$.

Thus, for any $b, b \in G$, we have

$$
g^{\prime}(b)=\left(\left(d g^{\prime}\right) e_{1}, \cdots,\left(d g^{\prime}\right) e_{n},\left(d g^{\prime}\right) J e_{1}, \cdots,\left(d g^{\prime}\right) J e_{n}\right),
$$

and hence, $\left(d g^{\prime}\right) J=J\left(d g^{\prime}\right)$, because $g^{\prime}(b) \in G$.

Therefore, we can conclude that $G$ acts transitively on $M$ as a group of automorphisms of $(M, J,\langle\rangle$,$) . This completes the proof of the latter$ half of Theorem B.

REMARK. Let $G$ be a transitive Lie group of automorphisms of an almost Hermitian manifold $(M, J,<\rangle$,$) . Then, the corresponding skew-$ symmetric tensor field $T$ of type $(1,2)$ on $M$ is $G$-invariant.

\section{The automorphism group of the maximum dimension.}

Let $(M, J,\langle\rangle$,$) be an 2 n$-dimensional almost Hermitian manifold of the automorphism group, say, $A(M)=A(M, J,\langle\rangle$,$) , of the maximum dimension,$ $n(n+2)$. Then, it follows that $A(M)$ acts transitively on $M$ (cf. [3], [4]). The linear isotropy group at a point $x$ of $A(M)$ may be considered as the unitary group $U(\mathrm{n})$, since $\operatorname{dim} A(M)=n(n+2)$ and $\operatorname{dim} M=2 n$. So it contains a 1-parameter group exp $(\sqrt{-1} t) I$, and in particular, the element, $-I$, where $I$ is the identity operator. If a tensor field $K$ of type $(r, s)$ is invariant by $A(M)$, then $K=0$ if $r+s$ is odd, since $-I$ maps $K$ into $(-1)^{r+s} K$. Since $(M, J,\langle\rangle$,$) is homogeneous, by Theorem \mathrm{B}$, there exists a skew-symmetric tensor field $T$ of type $(1,2)$ which corresponds to $A(M)$. Thus, by the above arguments, $T$ must vanish, since $T$ is invariant by $A(M)$. Therefore, in this case, $(M, J,\langle\rangle$,$) is a locally symmetric Kaehlerian manifold by (A),$ (B) and (C) in Theorem B. Furthermore, S. Tanno (cf. [4]) has proved that 
$(M, J,\langle\rangle$,$) is simply connected and is one of complex space forms of dimen-$ sion $n$.

Remark. Let $\left(S^{6},\langle,\rangle_{0}\right)$ be a standard 6-dimensional sphere in a 7 dimensional Euclidean space $\boldsymbol{E}^{7}$. Then, as is well known, $\left(S^{6},\langle,\rangle_{0}\right)$ is a space of positive constant curvature and $I\left(S^{6},\langle,\rangle_{0}\right)$ (the group of isometries of $\left.\left(S^{6},\langle,\rangle_{0}\right)\right)=O(7)$. In this case, $S^{6}=O(7) / O(6)$ as corresponding left coset space.

On the other hand, T. Fukami and S. ishihara (cf. [2]) showed that there exists a non-Kaehlerian, almost Tachibana (or nearly Kaehlerian) structure, say, $\left(J_{0},\langle,\rangle_{0}\right)$, on $S^{6}$. With respect to this structure $\left(J_{0},\langle,\rangle_{0}\right)$, the group of automorphisms $A\left(S^{6}, J_{0},\langle,\rangle_{0}\right)$ coincides with $G_{2}$ (cf. [2], [4]), and $S^{6}=G_{2} / S U(3)$ as corresponding left coset space. In this case, we can easily see that the expression $S^{6}=G_{2} / S U(3)$ corresponds to the tensor field $T(X)$ $Y=(1 / 2)\left(J_{0} \stackrel{0}{\nabla}_{X} J_{0}\right) Y$, where $\stackrel{0}{\nabla}$ denotes the Riemannian connection on $S^{6}$ with respect to $\langle,\rangle_{0}$.

\section{References}

[1] W. Ambrose and I. M. Singer: On homogeneous Riemannian manifolds, Duke Math. J., 25 (1958), 647-669.

[2] T. FUKAMI and S. IshiHARA: Almost Hermitian structure on $S^{6}$, Tôhoku Math. J., 7 (1955), 151-156.

[3] S. Kobayashi and K. NomizU: Foundations of differential geometry, Vol. I, II, Interscience, New York, 1963, 1969.

[4] S. TANNO: The automorphism groups of almost Hermitian manifolds, Trans. Amer. Math. Soc., 137 (1969), 269-275.

Faculty of Science Niigata University Niigata, Japan 